\title{
Distribution of the Largest Strong Goldbach Numbers Generated by Primes
}

\author{
Pingyuan Zhou ${ }^{1} \&$ Rong Ao ${ }^{2}$ \\ ${ }^{1}$ Beiyuan 35-210, Chengdu University of Technology, Chengdu, Sichuan, China \\ 210 Oak Grove St. Ottawa, Ontario K2G6R4, Canada \\ Correspondence: Pingyuan Zhou, Beiyuan 35-210, Chengdu University of Technology, Sichuan 610059, China. \\ E-mail: pingyuanzhou49@163.com
}

Received: May 21, 2018 Accepted: June 4, 2018 Online Published: June 26, 2018

doi:10.5539/jmr.v10n5p1 URL: https://doi.org/10.5539/jmr.v10n5p1

\begin{abstract}
Using the first 4000000 primes to find $L_{n}$, the largest strong Goldbach number generated by the $n$-th prime $P_{n}$, we generalize a proposition in our previous work (Zhou 2017) and propose that $L_{n} \approx 2 P_{n}$ and $L_{n} / 2 P_{n}<1$ for sufficiently large $P_{n}$ but the limit of $L_{n} / 2 P_{n}$ as $n \rightarrow \infty$ is $1, L_{n} \approx P_{n}+n \log n$ and $L_{n} /\left(P_{n}+n \log n\right)>1$ for sufficiently large $P_{n}$ but the limit of $L_{n} /\left(P_{n}+n \log n\right)$ as $n \rightarrow \infty$ is 1 . There are five corollaries of the generalized proposition for getting $L_{n} \rightarrow \infty$ as $n \rightarrow \infty$, which is equivalent to Goldbach's conjecture. If every step in distribution curve of $L_{n}$ is called a Goldbach step, a study on the ratio of width to height for Goldbach steps supports the existence of above two limits but a study on distribution of Goldbach steps supports an estimation that $Q(n) \approx(1+1 / \log \log n) n / \log n$ and the limit of $Q(n) /((1+1 / \log \log n) n / \log n)$ as $n \rightarrow \infty$ is 1 , where $Q(n)$ is the number of Goldbach steps, from which we may expect there are infinitely many Goldbach steps to imply Goldbach's conjecture.
\end{abstract}

Keywords: prime, largest strong Goldbach number, numerical evidence, Goldbach step, Goldbach's conjecture

2010 Mathematics Subject Classification: 11A41, 11B99

\section{Introduction}

In our previous work (Zhou 2017), we moved beyond traditional definition of Goldbach number (Montgomery, \& Vaughan, 1975; $\mathrm{Li}$ 1999; Lu 2010) by introducing three new definitions. First, $G_{n}=p+q$ is defined as a Goldbach number generated by the $n$-th prime $P_{n}$ for $n \geq 2$ if $p$ and $q$ are two odd primes not greater than $P_{n}$ (although $G_{n}$ is a number, there is a sequence $\left(G_{n}\right)$, for example, $\left(G_{5}\right)$ generated by $P_{5}=11$ is $(6,8,10,12,14,16,18,22)$ in which every term is a Goldbach number generated by $P_{5}$ ). Second, $G_{n}$ is defined as a strong Goldbach number generated by $P_{n}$ and written as $S_{n}$ if all even numbers from 6 to $G_{n}$ are Goldbach numbers generated by $P_{n}$ (although $S_{n}$ is a number, there is a sequence $\left(S_{n}\right)$, for example, $\left(S_{5}\right)$ generated by $P_{5}=11$ is $(6,8,10,12,14,16,18)$ in which every term is a strong Goldbach number generated by $P_{5}$ ). Third, $S_{n}$ is defined as the largest strong Goldbach number generated by $P_{n}$ and written as $L_{n}$ if $S_{n}+2$ is not a Goldbach number generated by $P_{n}$ ( here $L_{n}$ is always a number, for example, $L_{5}=18$ ). Proposition 3.2 in our work (Zhou 2017) proposed that $L_{n}-P_{n} \approx n \log n$, or equivalently $L_{n} \approx P_{n}+n \log n$ for all $P_{n}$. In this paper, we generalized the proposition based on the result of numerical calculation using the first 4000000 primes and provided a different approach for analyzing Goldbach's conjecture. We chose 4000000 because it is large enough to show tendency of $L_{n}$, yet small enough for our algorithm to generate data reasonably quickly. We examined the ratio of width to height for Goldbach steps and found an alternative approach to lead to the two limits in the generalized proposition. By studying distribution of Goldbach steps, we obtained an estimation such that $Q(n) \approx(1+1 / \log \log n) n / \log n$ and the limit of $Q(n) /((1+1 / \log \log$ $n) n / \log n)$ as $n \rightarrow \infty$ is 1 , where $Q(n)$ is the number of Goldbach steps.

\section{Distribution of the Largest Strong Goldbach Numbers Generated by Sufficiently Large Primes}

By definition of $L_{n}$, we have the following observations for sufficiently large primes.

Observation 2.1. Distribution of the ratio $L_{n} / 2 P_{n}$ for $10000 \leq n \leq 4000000$.

Figure 1 shows distribution of the ratio $L_{n} / 2 P_{n}$ for $10000 \leq n \leq 4000000$. From the distribution we see there is the same property such that $L_{n} / 2 P_{n} \approx 1$ and $L_{n} / 2 P_{n}<1$ for these primes but there is an obvious and stable increasing tendency, so one may estimate the limit of $L_{n} / 2 P_{n}$ as $n \rightarrow \infty$ is 1 , which reflects the fact that $2 P_{n}$ is the largest Goldbach number generated by $P_{n}$ (Zhou 2017). 


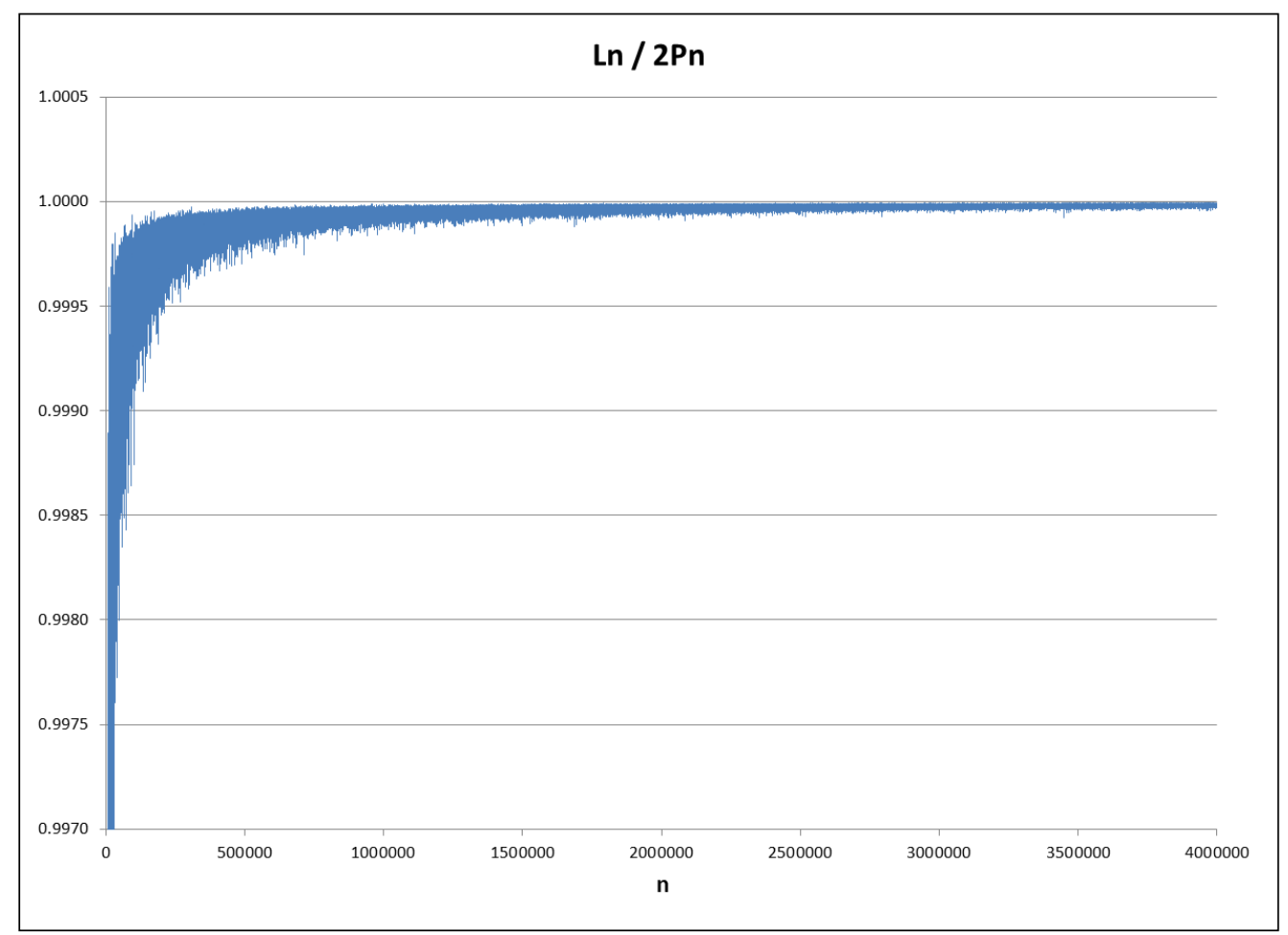

Figure 1. Distribution of the ratio $L_{n} / 2 P_{n}$ for $10000 \leq n \leq 4000000$

Observation 2.2. Distribution of the ratio $L_{n} /\left(P_{n}+n \log n\right)$ for $10000 \leq n \leq 4000000$.

Figure 2 shows distribution of the ratio $L_{n} /\left(P_{n}+n \log n\right)$ for $10000 \leq n \leq 4000000$. From the distribution we see there is the same property such that $L_{n} /\left(P_{n}+n \log n\right) \approx 1$ and $L_{n} /\left(P_{n}+n \log n\right)>1$ for these primes but there is an obvious and stable decreasing tendency, so one may estimate the limit of $L_{n} /\left(P_{n}+n \log n\right)$ as $n \rightarrow \infty$ is 1 .

Based on the above observations, we have the following proposition to lead to Goldbach's conjecture if the proposition is proven.

Proposition 2.3. $L_{n} \approx 2 P_{n}$ and $L_{n} / 2 P_{n}<1$ for sufficiently large $P_{n}$ but the limit of $L_{n} / 2 P_{n}$ as $n \rightarrow \infty$ is $1, L_{n} \approx P_{n}+n$ log $n$ and $L_{n} /\left(P_{n}+n \log n\right)>1$ for sufficiently large $P_{n}$ but the limit of $L_{n} /\left(P_{n}+n \log n\right)$ as $n \rightarrow \infty$ is 1 .

Remark 2.4. Proposition 2.3 is a generalization of our Proposition 3.2 (Zhou 2017). Any of the following five approaches would lead to $L_{n} \rightarrow \infty$ as $n \rightarrow \infty$, which is equivalent to Goldbach's conjecture (Zhou 2017).

Corollary 2.5. If $L_{n} \approx 2 P_{n}$ as $n \rightarrow \infty$, then $L_{n} \rightarrow \infty$ as $n \rightarrow \infty$.

Proof. Since $2 P_{n} \rightarrow \infty$ as $n \rightarrow \infty$ and $L_{n} \approx 2 P_{n}$ as $n \rightarrow \infty$, replace $2 P_{n}$ with $L_{n}$, so $L_{n} \rightarrow \infty$ as $n \rightarrow \infty$. Thus the corollary holds.

Corollary 2.6. If the limit of $L_{n} / 2 P_{n}$ as $n \rightarrow \infty$ is 1 , then $L_{n} \rightarrow \infty$ as $n \rightarrow \infty$.

Proof. Since $2 P_{n} \rightarrow \infty$ as $n \rightarrow \infty$ and $L_{n} / 2 P_{n} \rightarrow 1$ as $n \rightarrow \infty$, replace $2 P_{n}$ with $L_{n}$, so $L_{n} \rightarrow \infty$ as $n \rightarrow \infty$. Thus the corollary holds.

Corollary 2.7. If $L_{n} \approx P_{n}+n \log n$ as $n \rightarrow \infty$, then $L_{n} \rightarrow \infty$ as $n \rightarrow \infty$.

Proof. It has been proven in our work (Zhou 2017).

Corollary 2.8. If $L_{n} /\left(P_{n}+n \log n\right)>1$ as $n \rightarrow \infty$, then $L_{n} \rightarrow \infty$ as $n \rightarrow \infty$.

Proof. Since $P_{n}+n \log n \rightarrow \infty$ as $n \rightarrow \infty$ and $L_{n}>P_{n}+n \log n$ as $n \rightarrow \infty$, we have $L_{n} \rightarrow \infty$ as $n \rightarrow \infty$. Thus the corollary holds.

Corollary 2.9. If the limit of $L_{n} /\left(P_{n}+n \log n\right)$ as $n \rightarrow \infty$ is 1 , then $L_{n} \rightarrow \infty$ as $n \rightarrow \infty$.

Proof. Since $P_{n}+n \log n \rightarrow \infty$ as $n \rightarrow \infty$ and $L_{n} /\left(P_{n}+n \log n\right) \rightarrow 1$ as $n \rightarrow \infty$, replace $P_{n}+n \log n$ with $L_{n}$, so $L_{n} \rightarrow \infty$ as $n \rightarrow \infty$. Thus the corollary holds. 


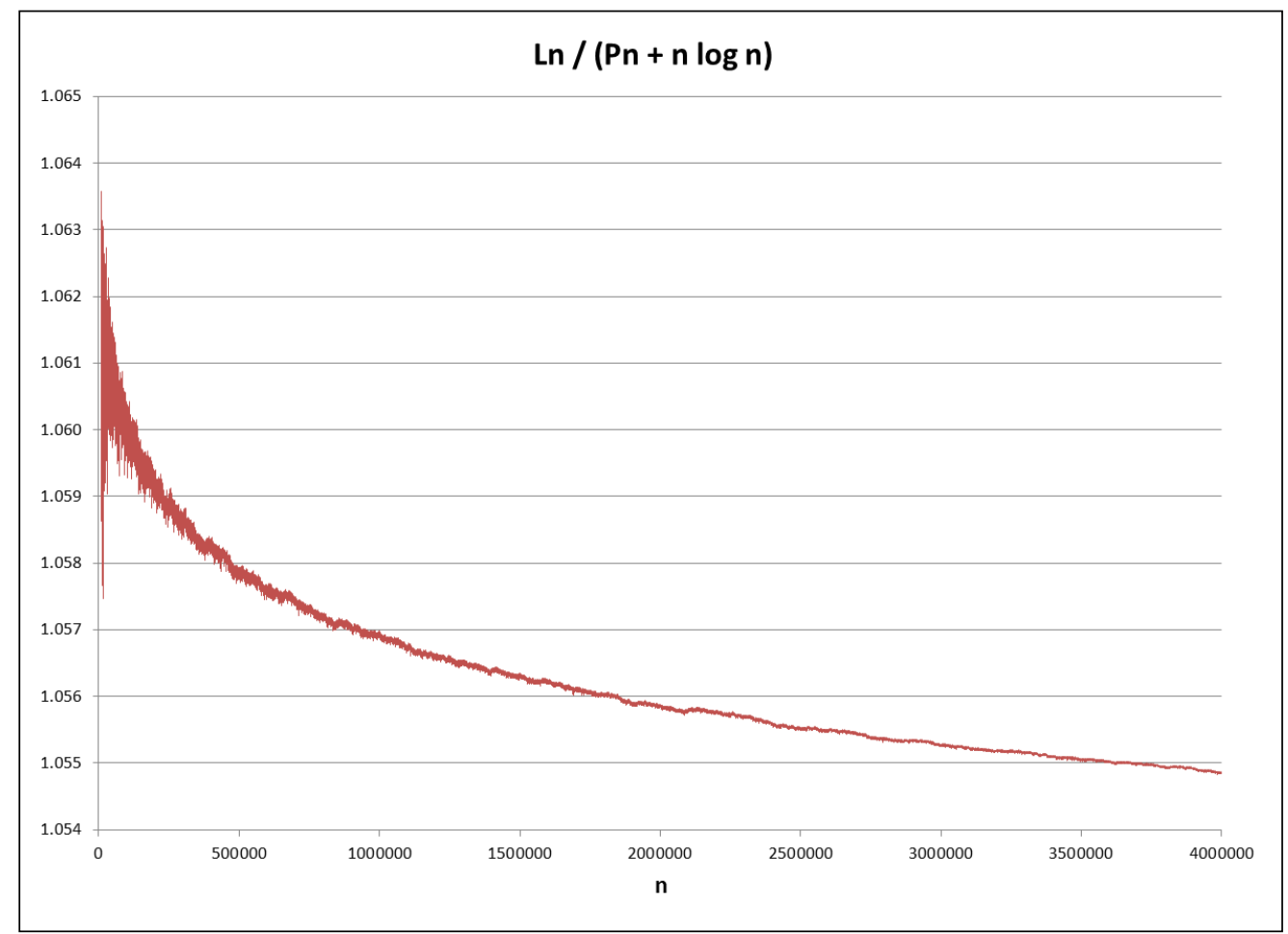

Figure 2. Distribution of the ratio $L_{n} /\left(P_{n}+n \log n\right)$ for $10000 \leq n \leq 4000000$

Our algorithm generated the following data for Figure 1 and Figure 2 at $n=10000,100000,1000000,2000000$, 3000000, 4000000: $L_{n} / 2 P_{n}=0.99746011,0.99958221,0.99997443,0.99996866,0.99997855,0.99998341$, and $L_{n} /\left(P_{n}+\right.$ $n \log n)=1.06144108,1.06011031,1.05697891,1.05585627,1.05526880,1.05485514$. It is obvious that $L_{n}$ is closer to $2 P_{n}$ than $P_{n}+n \log n$, but we will use both $2 P_{n}$ and $P_{n}+n \log n$ to study the ratio of width to height for steps in the distribution curve of $L_{n}$.

\section{Distribution of the Ratio of Width to Height for Goldbach Steps}

It could be derived from the definition of strong Goldbach number that if $S_{n}$ is a strong Goldbach number generated by $P_{n}$, then $S_{n}$ is also a strong Goldbach number generated by $P_{n+k}$ for $k \geq 1$ (Zhou 2017). Thus we have $L_{n} \leq L_{n+1}$ for any given $P_{n}$ so that the distribution of the largest strong Goldbach numbers generated by primes is a step-shaped curve growing without bound as Figure 3 shows.

Definition 3.1. Every step in the distribution curve of the largest strong Goldbach numbers $L_{n}$ generated by primes $P_{n}$ for $n \geq 2$ is called a Goldbach step.

Definition 3.2. For a given Goldbach step, $W$ is called width of the Goldbach step if $W=n_{2}-n_{1}$, where $n_{1}$ is $n$-value at the beginning of the Goldbach step and $n_{2}$ is $n$-value at the beginning of next Goldbach step.

Definition 3.3. For a given Goldbach step, $H$ is called height of the Goldbach step if $H=L_{n} \log L_{n}$, where $L_{n}$ is generated by $P_{n}$ with $n=n_{1}$ being $n$-value at the beginning of the Goldbach step.

According to Definition 3.2 and Definition 3.3, the ratio of width to height for a given Goldbach step is $W / H=\left(\left(n_{2}-\right.\right.$ $\left.\left.n_{1}\right) \log L_{n}\right) / L_{n}$. Figure 4 shows the distribution of $W / H=\left(\left(n_{2}-n_{1}\right) \log L_{n}\right) / L_{n}$ for $2 \leq n \leq 4000000$, Figure 5 shows the distribution of $\left(\left(n_{2}-n_{1}\right) \log 2 P_{n}\right) / 2 P_{n}$ for $2 \leq n \leq 4000000$ and Figure 6 shows the distribution of $\left(\left(n_{2}-n_{1}\right) \log \left(P_{n}+n\right.\right.$ $\log n)) /\left(P_{n}+n \log n\right)$ for $2 \leq n \leq 4000000$. Comparing Figure 5 and Figure 6 with Figure 4 respectively, we see that the figures are almost identical. By Figure 4, Figure 5 and Figure 6, it is obvious that $W / H \approx\left(\left(n_{2}-n_{1}\right) \log 2 P_{n}\right) / 2 P_{n}$ and $W / H$ $\approx\left(\left(n_{2}-n_{1}\right) \log \left(P_{n}+n \log n\right)\right) /\left(P_{n}+n \log n\right)$ but there is a tendency such that the limit of $\left(\left(n_{2}-n_{1}\right) \log L_{n}\right) / L_{n}$ as $n \rightarrow \infty$ is 0 . Therefore, one may expect that if the limit of $\left(\left(n_{2}-n_{1}\right) \log L_{n}\right) / L_{n}$ as $n \rightarrow \infty$ is 0 then $n_{2}-n_{1}$ must be smaller than $L_{n} / \log L_{n}$ for any given Goldbach step so that there is always a new and higher Goldbach step to follow any given Goldbach step and Goldbach's conjecture is true. 


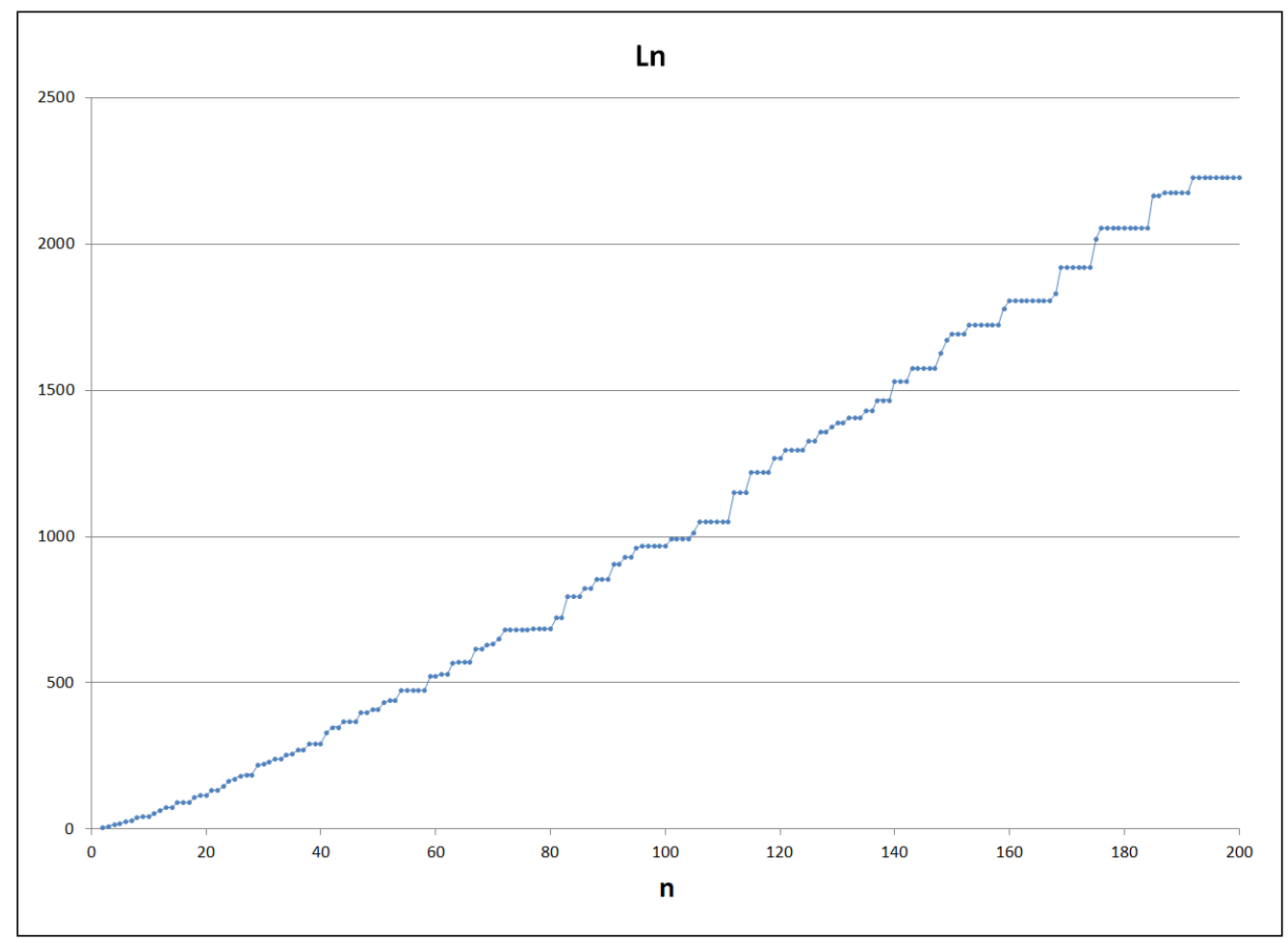

Figure 3. Step-shaped distribution of $L_{n}$ for $2 \leq n \leq 200$

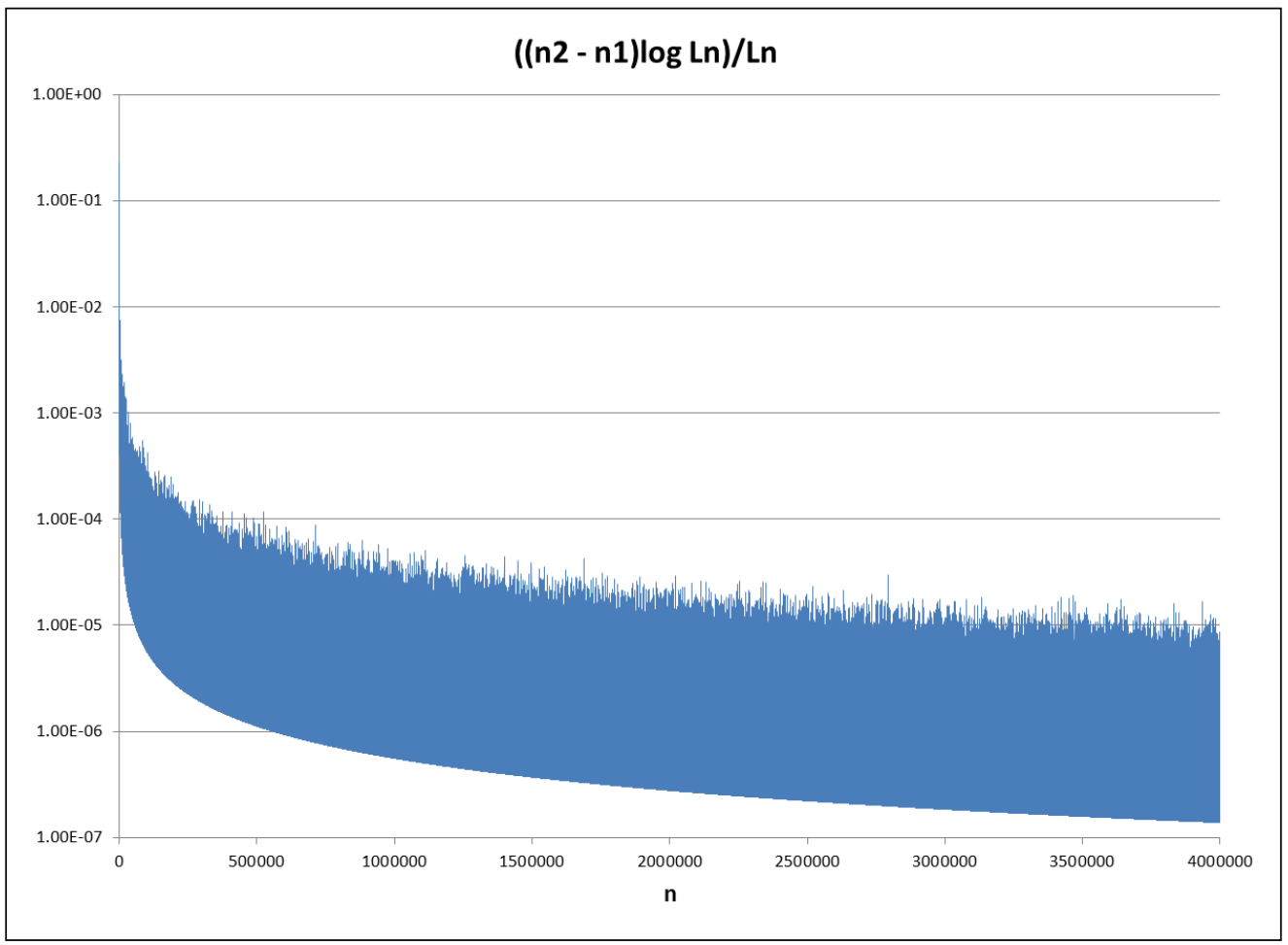

Figure 4. Distribution of $\left(\left(n_{2}-n_{1}\right) \log L_{n}\right) / L_{n}$ for $2 \leq n \leq 4000000$ 
In order to prove the limit of $\left(\left(n_{2}-n_{1}\right) \log L_{n}\right) / L_{n}$ as $n \rightarrow \infty$ is 0 , we obtain a set $\{A, B, C\}$, where $A=\left(\left(n_{2}-n_{1}\right) \log \right.$ $\left.2 P_{n}\right) / 2 P_{n}, B=\left(\left(n_{2}-n_{1}\right) \log L_{n}\right) / L_{n}$ and $C=\left(\left(n_{2}-n_{1}\right) \log \left(P_{n}+n \log n\right)\right) /\left(P_{n}+n \log n\right)$. If it is proven that $A$ is lower bound of the set and the limit of $\left(\left(n_{2}-n_{1}\right) \log 2 P_{n}\right) / 2 P_{n}$ as $n \rightarrow \infty$ is 0 but $C$ is upper bound of the set and the limit of $\left(\left(n_{2}\right.\right.$ $\left.\left.-n_{1}\right) \log \left(P_{n}+n \log n\right)\right) /\left(P_{n}+n \log n\right)$ as $n \rightarrow \infty$ is 0 , then the limit of $B=\left(\left(n_{2}-n_{1}\right) \log L_{n}\right) / L_{n}$ as $n \rightarrow \infty$ is 0 .

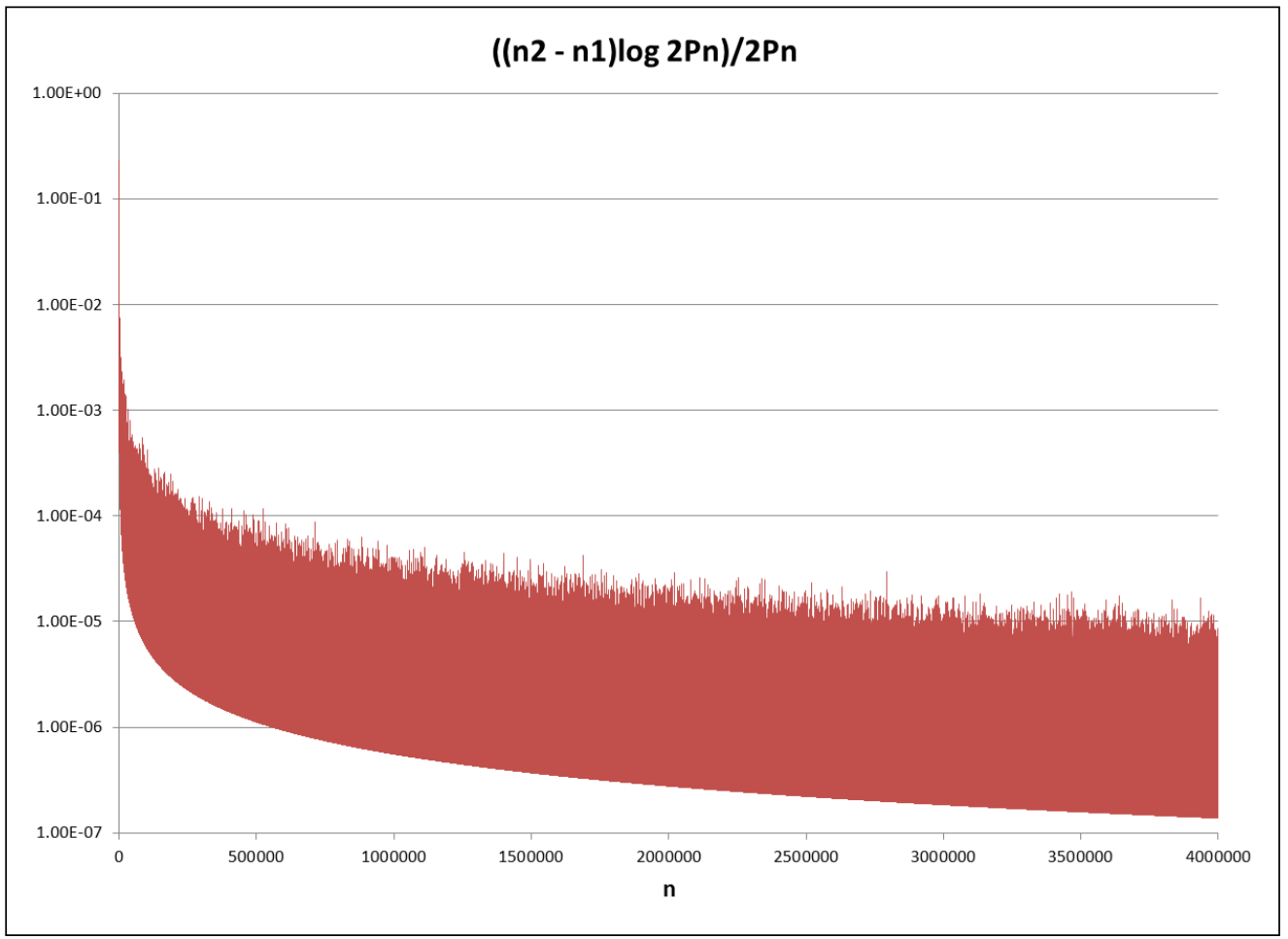

Figure 5. Distribution of $\left(\left(n_{2}-n_{1}\right) \log 2 P_{n}\right) / 2 P_{n}$ for $2 \leq n \leq 4000000$

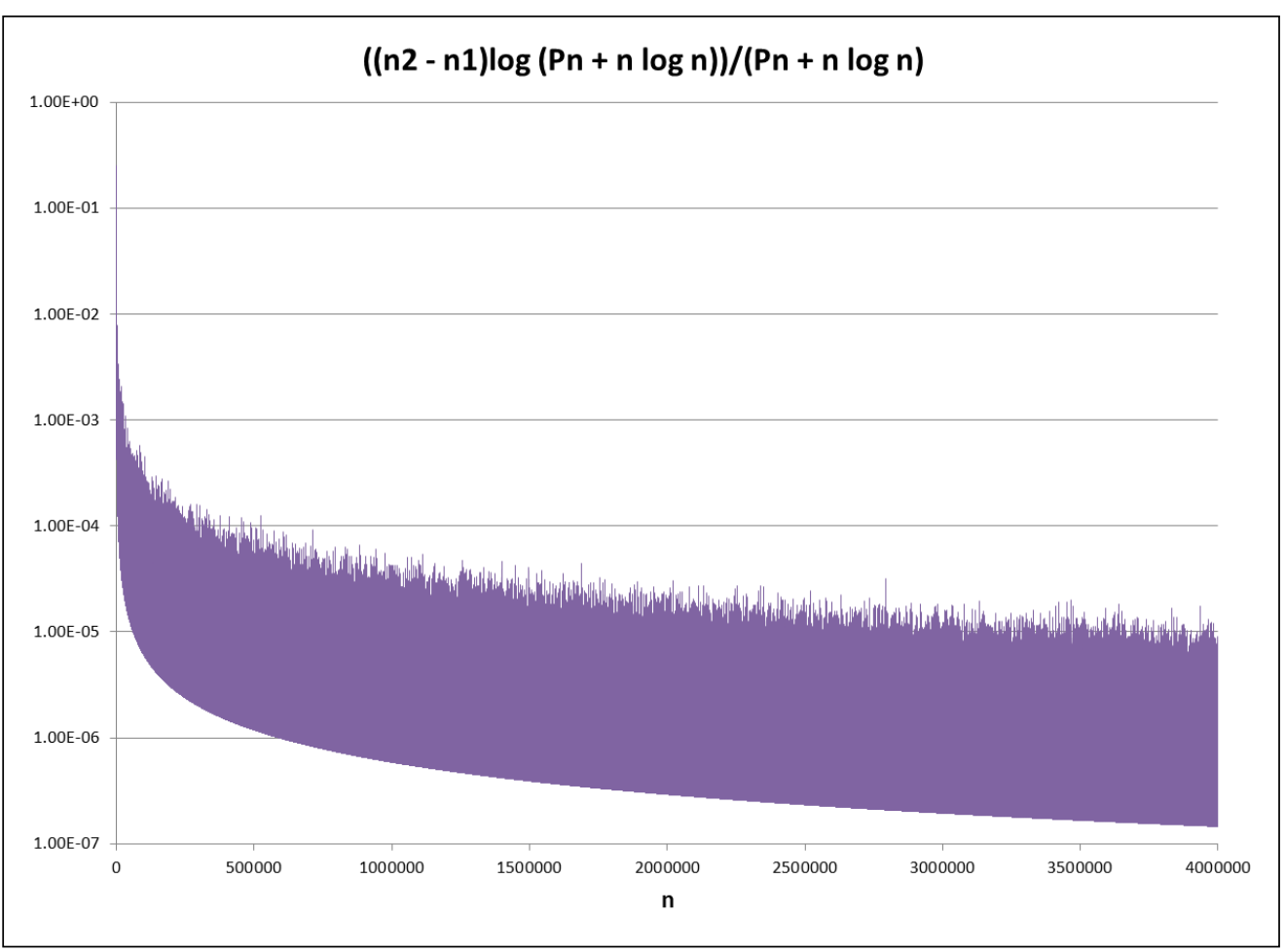

Figure 6. Distribution of $\left(\left(n_{2}-n_{1}\right) \log \left(P_{n}+n \log n\right)\right) /\left(P_{n}+n \log n\right)$ for $2 \leq n \leq 4000000$ 
Suppose it has been proven that the limit of $B=\left(\left(n_{2}-n_{1}\right) \log L_{n}\right) / L_{n}$ as $n \rightarrow \infty$ is 0 . Since the limit of $A / B$ as $n \rightarrow \infty$ is 1 but $A / B=\left(L_{n} / 2 P_{n}\right)\left(\log 2 P_{n}\right) / \log L_{n}$, if the limit of $\left(\log 2 P_{n}\right) / \log L_{n}$ as $n \rightarrow \infty$ is 1 then the limit of $L_{n} / 2 P_{n}$ as $n \rightarrow \infty$ is 1 . Since the limit of $C / B$ as $n \rightarrow \infty$ is 1 but $C / B=\left(L_{n} /\left(P_{n}+n \log n\right)\right)\left(\log \left(P_{n}+n \log n\right)\right) / \log L_{n}$, if the limit of $\left(\log \left(P_{n}+n\right.\right.$ $\log n)) / \log L_{n}$ as $n \rightarrow \infty$ is 1 then the limit of $L_{n} /\left(P_{n}+n \log n\right)$ as $n \rightarrow \infty$ is 1 . Thus above results are equivalent to the two limits in Proposition 2.3.

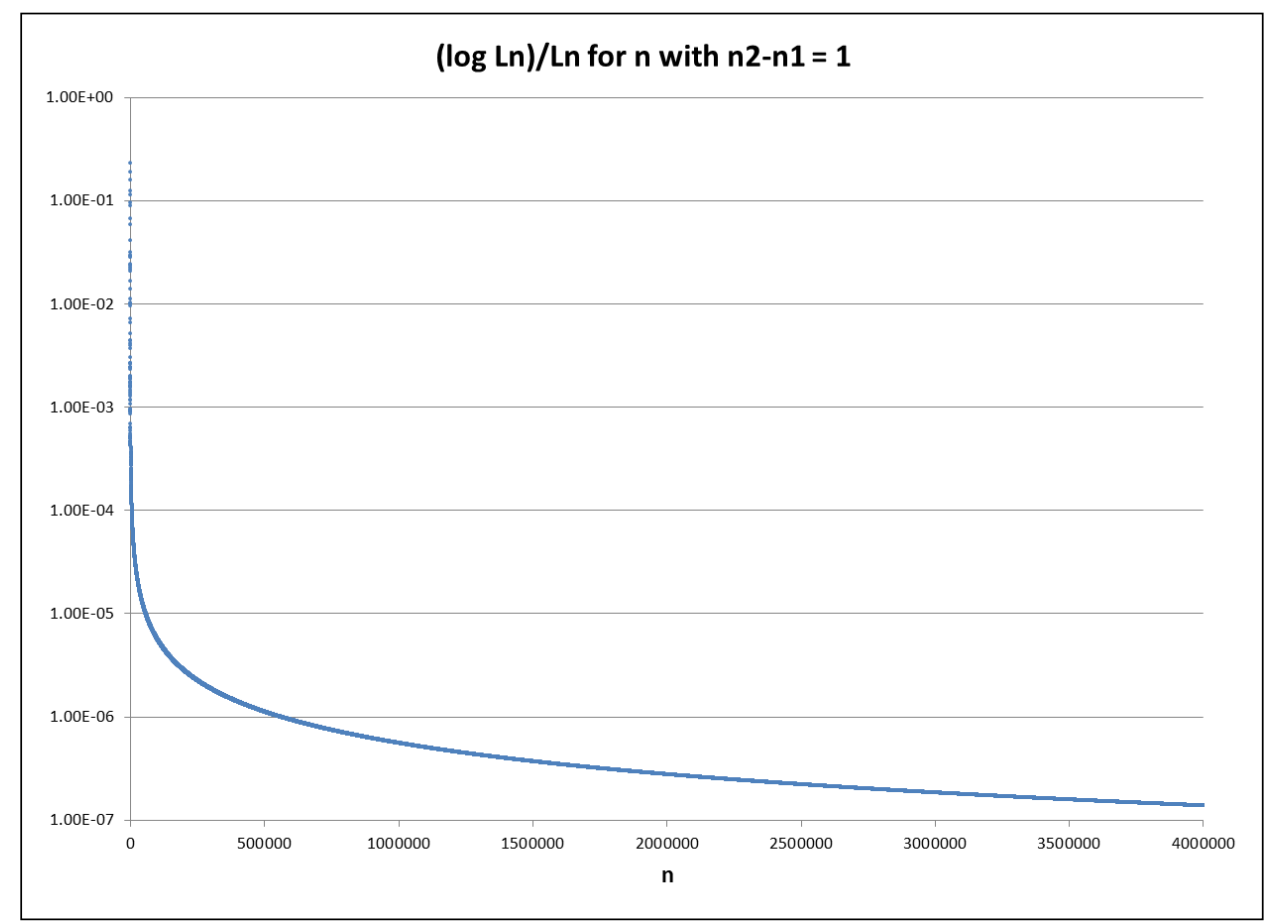

Figure 7. Distribution of $\left(\log L_{n}\right) / L_{n}$ for $n$ with $n_{2}-n_{1}=1$ for $2 \leq n \leq 4000000$

Further, we study bottom curves in Figure 4, Figure 5 and Figure 6 because these curves look smooth and there is the same tendency such that the three curves will approach 0 as $n$ goes to infinity. First, we should consider the bottom curve of $A=\left(\left(n_{2}-n_{1}\right) \log 2 P_{n}\right) / 2 P_{n}$ in Figure 5 and we are sure that the curve is formed by minimum values of $A$. Let $n_{2}$ $-n_{1}=1$. Then we obtain $A_{\min }=\left(\log 2 P_{m}\right) / 2 P_{m}$ if $m$ denotes the $n$-value at which $\left(\log 2 P_{n}\right) / 2 P_{n}$ is a minimum value of $A$. It is obvious that $2 P_{m} / \log 2 P_{m}$ is the number of primes among all integers from 1 to $2 P_{m}$, therefore, $1 / A_{\text {min }}=2 P_{m} / \log 2 P_{m}$ is just the number of primes not greater than $2 P_{m}$. Similarly, we have $1 / C_{\min }=\left(P_{m}+m \log m\right) / \log \left(P_{m}+m \log m\right)$ if $m$ denotes the $n$-value at which $\left(\log \left(P_{n}+n \log n\right)\right) /\left(P_{n}+n \log n\right)$ is a minimum value of $C$ in Figure 6 but we have $1 / B_{\text {min }}$ $=L_{m} / \log L_{m}$ if $m$ denotes the $n$-value at which $\left(\log L_{n}\right) / L_{n}$ is a minimum value of $B$ in Figure 4 . Finally, Figure 7 shows the distribution of $\left(\log L_{n}\right) / L_{n}$ for $n$ with $n_{2}-n_{1}=1$ for $2 \leq n \leq 4000000$, that is, the distribution of $B_{\min }=\left(\log L_{m}\right) / L_{m}$ for $2 \leq n \leq 4000000$. Comparing Figure 7 with Figure 4, we see the curve in Figure 7 is just the bottom curve in Figure 4 .

\section{Distribution of Goldbach Steps}

Let $Q(n)$ denote the number of Goldbach steps formed by the first $n$ primes $P_{n}$ ( the number is just the number of $n_{1}$ among the first $n$ primes $P_{n}$ ). Then we have the following observations.

Observation 4.1. Distribution of $Q(n)$ for $2 \leq n \leq 4000000$.

Figure 8 shows the distribution of $Q(n)$ for $2 \leq n \leq 4000000$, which is a curve with continuous growths. In the figure, another curve shows the distribution of $(1+1 / \log \log n) n / \log n$, in which $n / \log n$ denotes the number of primes among the first $n$ positive integers.

Observation 4.2. Distribution of the ratio $Q(n) /((1+1 / \log \log n) n / \log n)$ for $2 \leq n \leq 4000000$.

Figure 9 shows the distribution of $Q(n) /((1+1 / \log \log n) n / \log n)$ for $2 \leq n \leq 4000000$. From the distribution we see $Q(n) /((1+1 / \log \log n) n / \log n) \approx 1$ for these $n$-values and there is a decreasing tendency, so one may estimate the limit of $Q(n) /((1+1 / \log \log n) n / \log n)$ as $n \rightarrow \infty$ is 1 .

Based on the above two observations, we have the following proposition.

Proposition 4.3. $Q(n) \approx(1+1 / \log \log n) n / \log n$ and the limit of $Q(n) /((1+1 / \log \log n) n / \log n)$ as $n \rightarrow \infty$ is 1 . 


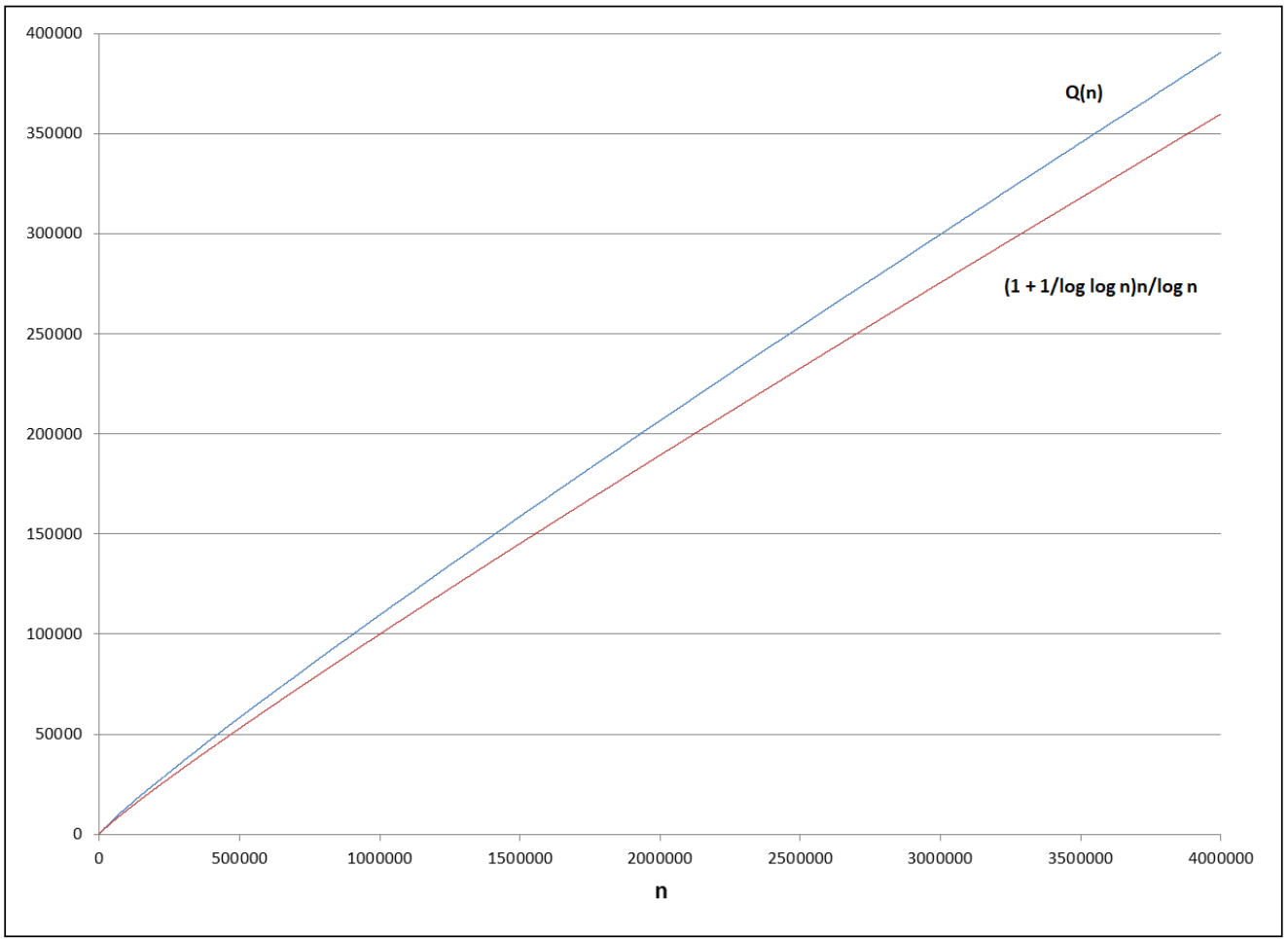

Figure 8. Distributions of $Q(n)$ and $(1+1 / \log \log n) n / \log n$ for $2 \leq n \leq 4000000$

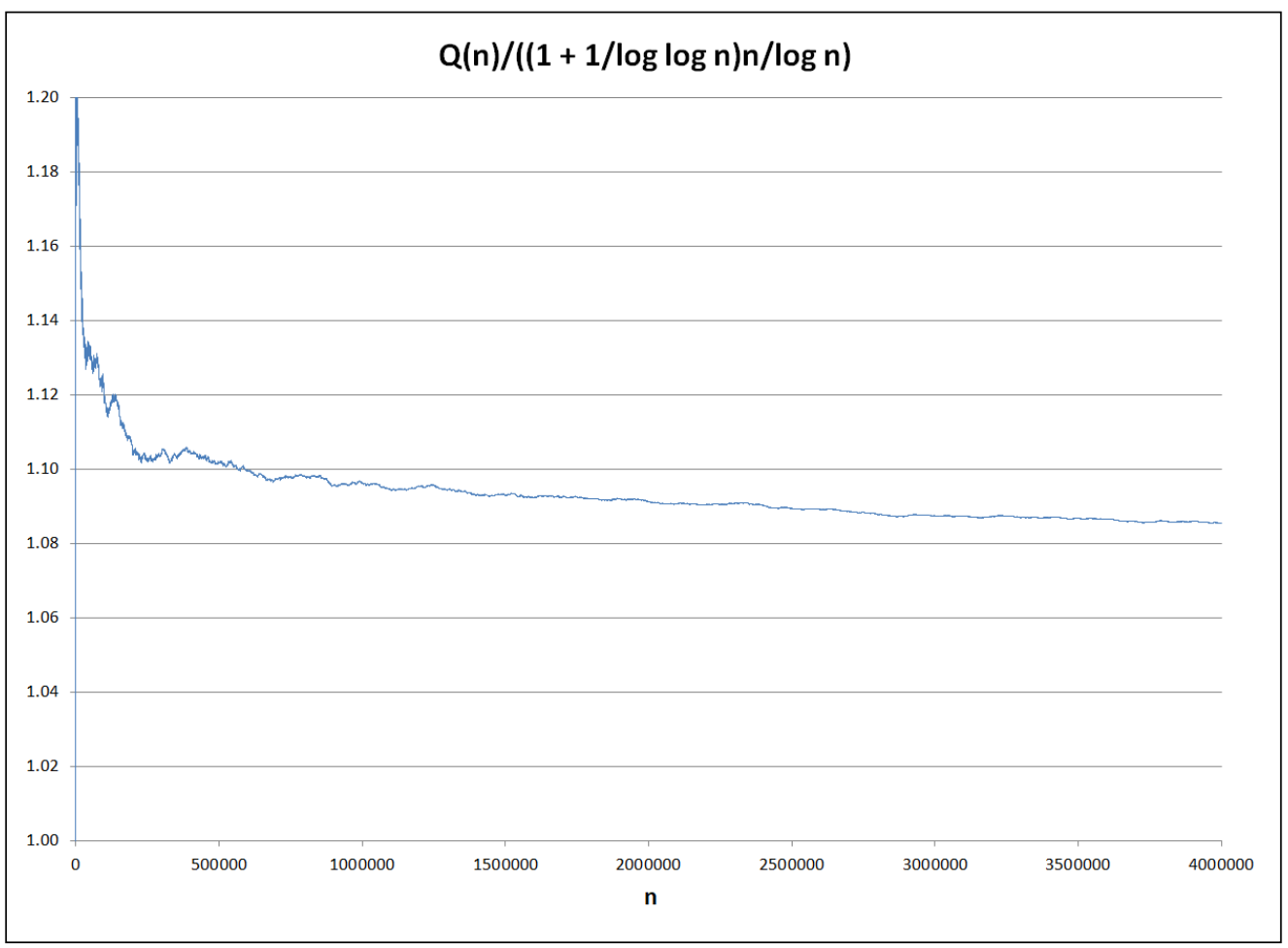

Figure 9. Distribution of the ratio $Q(n) /((1+1 / \log \log n) n / \log n)$ for $2 \leq n \leq 4000000$ 
Corollary 4.4. If $Q(n) \approx(1+1 / \log \log n) n / \log n$ as $n \rightarrow \infty$, then $L_{n} \rightarrow \infty$ as $n \rightarrow \infty$.

Proof. Since $(1+1 / \log \log n) n / \log n \rightarrow \infty$ as $n \rightarrow \infty$ and $Q(n) \approx(1+1 / \log \log n) n / \log n$ as $n \rightarrow \infty$, replace $(1+1 / \log$ $\log n) n / \log n$ with $Q(n)$, so $Q(n) \rightarrow \infty$ as $n \rightarrow \infty$. The result means there are infinitely many Goldbach steps, which obviously implies $L_{n} \rightarrow \infty$ as $n \rightarrow \infty$. Thus the corollary holds.

Corollary 4.5. If the limit of $Q(n) /((1+1 / \log \log n) n / \log n)$ as $n \rightarrow \infty$ is 1 , then $L_{n} \rightarrow \infty$ as $n \rightarrow \infty$.

Proof. Since $(1+1 / \log \log n) n / \log n \rightarrow \infty$ as $n \rightarrow \infty$ and $Q(n) /((1+1 / \log \log n) n / \log n) \rightarrow 1$ as $n \rightarrow \infty$, replace $(1+$ $1 / \log \log n) n / \log n$ with $Q(n)$, so $Q(n) \rightarrow \infty$ as $n \rightarrow \infty$. The result means there are infinitely many Goldbach steps, which obviously implies $L_{n} \rightarrow \infty$ as $n \rightarrow \infty$. Thus the corollary holds.

Remark 4.6. If Proposition 4.3 is proven then there are infinitely many Goldbach steps to imply $L_{n} \rightarrow \infty$ as $n \rightarrow \infty$ by Corollary 4.4 or Corollary 4.5 , therefore, Goldbach's conjecture is true.

\section{Conclusion}

In this paper, we presented studies on distributions of $L_{n} / 2 P_{n}$ and $L_{n} /\left(P_{n}+n \log n\right)$, distribution of the ratio of width to height for Goldbach steps and distribution of Goldbach steps using numerical evidence for the first 4000000 primes. We obtained Proposition 2.3 which is a generalization of previous Proposition 3.2 (Zhou 2017). If any of the five approaches derived from Proposition 2.3 is proven, then Goldbach's conjecture is also proven true. By ratio of width to height for Goldbach steps, we assumed the limit of $B=\left(\left(n_{2}-n_{1}\right) \log L_{n}\right) / L_{n}$ as $n \rightarrow \infty$ is 0 , which will be equivalent to the limit of $L_{n} / 2 P_{n}$ as $n \rightarrow \infty$ being 1 and the limit of $L_{n} /\left(P_{n}+n \log n\right)$ as $n \rightarrow \infty$ being 1 in Proposition 2.3, if it is proven that the limit of $A=\left(\left(n_{2}-n_{1}\right) \log 2 P_{n}\right) / 2 P_{n}$ as $n \rightarrow \infty$ is 0 and the limit of $C=\left(\left(n_{2}-n_{1}\right) \log \left(P_{n}+n \log n\right)\right) /\left(P_{n}+n\right.$ $\log n)$ as $n \rightarrow \infty$ is 0 but $A$ and $C$ are lower and upper bounds of the set $\{A, B, C\}$. By distribution of Goldbach steps, we discovered a pattern that $Q(n) \approx(1+1 / \log \log n) n / \log n$ to support the existence of the limit of $Q(n) /((1+1 / \log \log$ $n) n / \log n)$ as $n \rightarrow \infty$ being 1, which means there may be infinitely many Goldbach steps to imply Goldbach's conjecture.

\section{Acknowledgments}

The authors would like to acknowledge editor and reviewer for their careful reading and valuable comments, and thank Yuming Chen for his helpful suggestions.

\section{References}

Li, H. (1999). The Exceptional Set of Goldbach Numbers. Quart J Math Oxford(2), 50, 471-482. https://doi.org/10.1093/qjmath/50.200.471

Lu, W. C. (2010). Exceptional set of Goldbach number. J Number Theory, 130(10), 2359-2392. https://doi.org/10.1016/j.jnt.2010.03.017

Montgomery, H. L., \& Vaughan, R. C. (1975). The exceptional set in Goldbach's problem. Acta Arith, 27, 353-370. https://doi.org/10.4064/aa-27-1-353-370

Zhou, P. (2017). Strong Goldbach Number in Goldbach's Problem. Journal of Mathematics Research, 9(6), 95-105. https://doi.org/10.5539/jmr.v9n6p95

\section{Copyrights}

Copyright for this article is retained by the author(s), with first publication rights granted to the journal.

This is an open-access article distributed under the terms and conditions of the Creative Commons Attribution license (http://creativecommons.org/licenses/by/4.0/). 